been predicted almost three decades earlier by Marcus ${ }^{5}$. Others then synthesized and characterized even more complex systems, for example, bearing three or more organic donors and acceptors on a common covalent frame ${ }^{6}$, or an organic donor tethered to an inorganic cluster ${ }^{7}$ with backbones varying in physical character from rigid to flexible. The critical role of the precursor conformation in exciplex formation has also been demonstrated previously, for example, in van der Auweraer, Gilbert and de Schryver's study of alkanes of varying lengths capped by donors and acceptors ${ }^{8}$. What is novel about the work of Verhoeven et al. is its clear demonstration of conformational change induced specifically by the intramolecular electron transfer.

For this electrostatically driven folding to follow light-induced electron transfer, several criteria must be met: first, an energetic driving force sufficiently strong to cause light-initiated electron transfer over large distances from the donor to the acceptor moiety; second, dispersion of the molecule of interest within a nonpolar solvent where solvation of the directly formed charge-transfer state is less stabilizing than the coulombic attraction between the charged sites; and third, a molecular backbone sufficiently rigid to be fully stretched in its preferred ground-state conformation, yet maintaining enough flexibility to fold in response to the interaction of the opposite charges. Although these criteria may not be applicable to all cases of conformational change, they may be relevant in other systems. For example, conformational gating of ion transport in proteins ${ }^{9}$ may be responsive to many of the same factors.

Marye Anne Fox is in the Department of Chemistry, University of Texas, Austin, Texas, USA.

1. Brouwer, A. M. et al. Chem. Phys. Lett. 186, 481-489 (1991).

2. Fox, M. A. \& Chanon, M. (eds) Photoinduced Electron Transfer Vols A-D (Elsevier, Amsterdam, 1988).

3. Miller, J. R., Calcaterra, L. T. \& Closs, G. L. J. Am. chem. Soc. 106, 3047-3049 (1984).

4. Calcaterra, L. T., Closs, G. L. \& Miller, J. R. J. Am. chem. Soc. 105, 670-672 (1983)

5. Marcus, R. A. J. chem. Phys. 24, 966 (1956)

6. Gust, D. \& Moore, T. A. Science 244, 35-41 (1989).

7. Resch, U. \& Fox, M. A. J. phys. Chem. 95, 6169 (1991).

8. van der Auweraer, M. Gilbert, A. \& de Schryver, F. C. van der Auweraer, M., Gilbert, A. \& de

. Hoffman, B. M., Ratner, M. A. \& Wallin, S. A. Adv. Chem 226. $125-146$ (1990).

\title{
Enemies doomed to associate
}

\section{Jared M. Diamond}

TAKE two species. If, when their distributions are mapped in chessboard fashion, they appear on different squares only, one might conclude that they are competitors; and if they consistently coexist on the same squares, one might think they are mutualists. But things are not so simple, because such patterns might well arise from the species' differing or coincident preferences for a particular habitat. That problem, for a long time a thorny one for ecological biogeographers, has been tackled by $\mathrm{T}$. Schoener and G. Adler (Am. Nat. 137, $669-692 ; 1991$ ), who have now come up with a highly effective way of separating the effects of species interactions on species distributions from the effects of habitat variables.

The distinction matters especially in studies of guilds of potentially competing species. Precisely because competitors must be ecologically similar, their shared habitat requirements lead indirectly towards coincident distributions - the opposite of the direct effect of competition itself, which may lead towards complementary distributions. For example, two competing species of freshwater duck will both tend to be absent on islands without fresh water but present on islands with fresh water. Not surprisingly, most statistically significant associations among bird species of the Bis- marck archipelago are positive, reflecting the familiar overwhelming effect of habitat requirements (M. Gilpin and J. Diamond, Oecologia 52, 75-84; 1982). One would therefore expect that, if this dominant trend towards positive associations due to shared habitat requirements could somehow be stripped away, stronger evidence of negative associations due to competition would be unmasked.

Schoener and Adler's technique combines two elements. The independent variables are 16 continuously varying ecological parameters likely to influence species distributions, such as island area, isolation, maximum altitude, habitat or foliage diversity, and amounts of vegetation at various heights above the ground. The dependent variables are species codistributions, expressed in multiway contingency tables of the presence or absence of particular species (for example whether a given island supports species $\mathrm{A}$ and $\mathrm{B}, \mathrm{A}$ but not B, B but not A, or neither). The database used for the test consists of published distributions of 40 species of diurnal lizards and resident birds on 52 Bahamian islands. In practice, Schoener and Adler construct two-, three- and four-way contingency tables (examining associations within sets of two to four species) for 23 guilds of lizard or bird species selected as being ecologically homogeneous (ground

\section{Hopalong}

ERRATIC hopping movements of water droplets on the warmed surface of solid cyclohexane provide much-sought evidence of microscopic forces at interfaces, report A. Steyer et al. (Phys. Rev. Lett. 68, 64-66; 1992). The droplets were condensing from a stream of water-saturated nitrogen, and the surface was maintained just below $6.68^{\circ} \mathrm{C}$, cyclohexane's melting point. The authors were surprised to find the growing drops move, rotate and even jump. The explanation for these phenomena is in the forces created at a drop's edge by surface tension, which elastically deform the substrate structure. The heat of condensation released as the drop grows is just enough to melt the solid locally, allowing the elastic strain to be released in a quick flick. If a drop is pinned to a surface defect, it is likely to spin rather than jump. Until now, these contact forces had been talked about, but had not been observed on rigid surfaces.

\section{Head gear}

THE single-headed version of myosin, myosin I, is known to bind directly to lipid molecules, unlike the more common double-headed myosin II. T. D. Pollard and colleagues (J. Cell Biol. 116, $367-376 ; 1992$ ) have used the gliding filament assay to demonstrate that myosin I, when bound to pure lipid bilayers, is able to activate the movement of actin filaments. The new results provide an explanation for how myosin I can support motility of certain cells and organelles, and they suggest a mechanism for how the molecule is targeted to plasma membranes in Acanthamoeba, Dictyostelium and some vertebrate epithelial cells.

\section{Milky ways}

THE well-known but poorly documented advantages of mothers' milk are given a firm basis in a UK study reported in this week's Lancet (339, 261-264; 1992). A. Lucas and collaborators show that preterm babies whose mother provided breast milk (either by breastfeeding or by a tube) for the early weeks of life had a substantial advantage in subsequent IQ at 7-8 years of age. The authors adjusted for various social and economic factors, but genetic factors and differences in parental behaviour cannot be completely excluded. Lucas et al. believe that their data provide a strong indication that human breast milk contains one or more components that influence neurodevelopment. One of the important implications for infant care would be the reintroduction of milk banks, now generally fallen out of use in Britain, by which premature and other babies can be fed milk donated by lactating mothers rather than infant formula. 
lizards, small insectivorous birds and seed-eating finches, for instance).

The first step in the analysis consists of logistic regression, for each species and independent variable, of that species: probability of occurrence on an island as a function of the variable. Such regressions are exemplified by graphs of 'incidence' (probability of occurrence) as a function of island area or species number. By an iterative procedure Schoener and Adler extract such species/habitat regressions plus the interactions within each combination of species (that is,

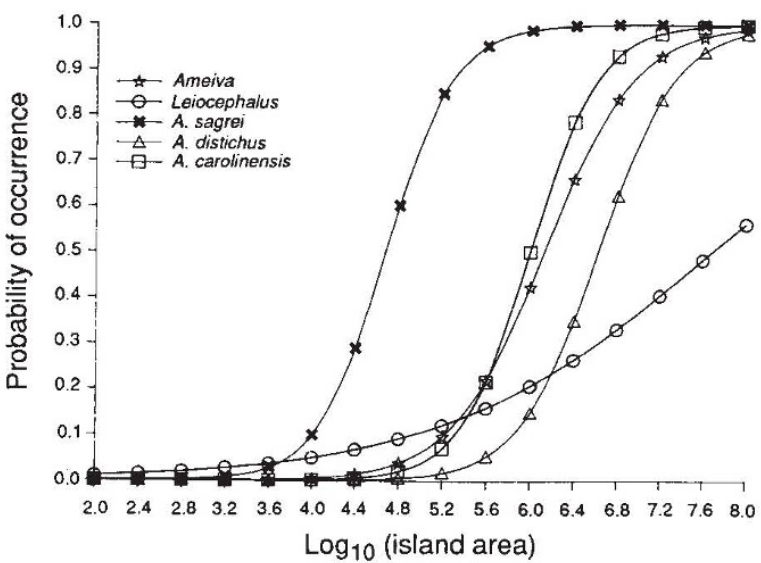

Logistic regression of the proportions of Bahamian islands in a given size class occupied by various lizard species, as a function of $\log _{10}$-transformed island area. That proportion of islands has been termed 'incidence'. The steeper the rise of the curve, the sharper in some sense is the threshold range of island sizes separating islands with and without the named species. (From T. Schoener and G. Adler Am. Nat. 137, 669-692; 1991.)

distributional overlap or avoidance not explained by the species/habitat regressions themselves). The authors stress that the application of this statistical technique to distributional data is new and warrants further testing.

The most striking conclusion is that, after correcting for the effects of habitat variables, the analysis tends to convert positive two-way associations between species (as observed in the raw distributional data) into negative associations. That is, although the direct interaction between the two species is actually negative (they tend to exclude each other), the interaction is masked in the raw data by the similar responses of both species to habitat variables. In effect, the species are enemies doomed to seek out the same types of sites. For instance, to return to the example of two competing duck species, both ducks would be absent from small islands without ponds, both would be present on islands large enough to contain different types of ponds, and only in the range of mediumsized islands with just a single pond would the ducks occupy different islands to the exclusion of each other.

These masking effects of habitat variables prove to be huge. As a result of stripping them away, the proportion of two-way species interactions that are negative increases from 24 to 67 per cent for lizards, and from 3 to 47 per cent for birds. In addition, interactions that are already negative in the raw data become even more strongly so after considering habitat variables.

To interpret the signs of these interactions, Schoener and Adler note that the negative interactions are likely to represent competition, because the species analysed were chosen to be members of the same guild. Examples include wellknown pairs of species that have provided textbook instances of complementary island distributions, such as the mockingbirds Mimus gundlachi and $M$. polyglottos or the lizards Anolis sagrei and the Leiocephalus group. The residual positive interactions may represent parallel responses of species to habitat variables not among those analysed by Schoener and Adler. So two hummingbird species are positively associated both in the raw data and after correction for habitat variables - but probably because distributions of both species are tied to flower abundance, not among the dependent variables measured.

Several other points emerge from the study. Three-way interactions are overwhelmingly negative, reflecting in part the importance of diffuse competition. Negative interactions are more common among lizard species than among bird species, possibly owing to birds' higher immigration rates, hence smaller populations and shorter population lifetimes. Raw habitat variables yield stronger trends than do compound variables produced by principal components analysis.

The main message, however, is that if one wants to test occurrence data for species interactions, one should control for habitat relations because of their huge masking effect. Biogeographers will now be stimulated to reanalyse the many published lists of species occurrences on islands (as well as on islandlike habitat patches) of known area, isolation and elevation. And field workers will be stimulated to measure a greater variety of habitat variables in their studies, for Schoener and Adler have now provided a recipe for taking such measurements into account

Jared $M$. Diamond is in the Department of Physiology, University of California Medical School, Los Angeles, California 90024, USA.

\section{Occult learning}

LAST week Daedalus proposed his 'Skygazer' space telescope. Its forty thousand photodetectors, dividing up the sky between them in fly's-eye fashion, will count all the photons hitting the telescope from the celestial sphere. With appropriate shielding from the Sun, Earth and Moon, it will be exquisitely, ultimately sensitive to the slightest variation of brightness from the rest of the sky.

The Skygazer will be ideal for locating stellar occultations. Daedalus estimates that in a galaxy of ten thousand million stars all wandering around, there must always be at least one star eclipsing another as seen from the Earth: yet (except in the special case of eclipsing binary stars) such an occultation has never been observed. This is not surprising. An occultation is quite undramatic, gives no warning, and cannot last much longer than a day. You would never spot it unless you knew where to look.

The Skygazer spacecraft will tell you. It watches all the sky all the time. Its ultimate photometric accuracy will instantly register the slight loss of light from that section of sky where one star is beginning to hide behind another. The detector whose count is changing will locate the region of loss to within one square degree of sky. An Earth-based Schmidt telescope could then be trained onto that region to find the occultation.

From the changing brightness and spectrum of this joint stellar image, useful information will be gained about the relative velocity, size, brightness, limb darkening and even shape of the protagonists. As the stars then separate, the shifting image of the occulted star as its light is deflected relativistically by the mass of the occulter, will give a measure of that mass. Occultations will be more frequent in some parts of the sky than in others, yielding statistical data on the density and velocity-distribution of stars in various regions of the galaxy.

Best of all, occultation offers about the only possible way of spotting the mysterious 'dark matter' that cosmologists insist must be lying about somewhere. Brown dwarfs, big planets, black clouds and general nonluminous celestial junk, will at last be detectable as they drift in front of luminous stars. If such dark objects have an atmosphere, or even a refractive index, informative lens-effects will further enrich their occultations. And should the long-feared impacting asteroid, or a spacecraft crammed with militant little green men, ever head menacingly towards the Earth, it should reveal itself by occulting background stars long before it could be detected on radar. 